\title{
Review Article \\ Role of Autophagy and Apoptosis in the Postinfluenza Bacterial Pneumonia
}

\author{
Zhen Qin, ${ }^{1}$ Yuan Yang, ${ }^{1}$ Hongren Wang, ${ }^{1}$ Jun Luo, ${ }^{1}$ Xiaojun Huang, \\ Jiangzhou You, ${ }^{1}$ Baoning Wang, ${ }^{1}$ and Mingyuan $\mathrm{Li}^{1,2}$ \\ ${ }^{1}$ Department of Microbiology, West China School of Preclinical and Forensic Medicine, Sichuan University, \\ Chengdu, Sichuan 610041, China \\ ${ }^{2}$ State Key Laboratory of Oral Diseases, Sichuan University, Chengdu, Sichuan 610041, China
}

Correspondence should be addressed to Mingyuan Li; lmy3985@sina.com

Received 14 January 2016; Revised 5 April 2016; Accepted 16 May 2016

Academic Editor: Kevin M. Coombs

Copyright (C) 2016 Zhen Qin et al. This is an open access article distributed under the Creative Commons Attribution License, which permits unrestricted use, distribution, and reproduction in any medium, provided the original work is properly cited.

The risk of influenza A virus (IAV) is more likely caused by secondary bacterial infections. During the past decades, a great amount of studies have been conducted on increased morbidity from secondary bacterial infections following influenza and provide an increasing number of explanations for the mechanisms underlying the infections. In this paper, we first review the recent research progress that IAV infection increased susceptibility to bacterial infection. We then propose an assumption that autophagy and apoptosis manipulation are beneficial to antagonize post-IAV bacterial infection and discuss the clinical significance.

\section{Introduction}

(1) Influenza A Virus and Secondary Bacterial Infection. It was reported that a pandemic influenza killed over 40 million people in 1918 [1, 2]. With the development of modern medicine and improvement of hygiene habits, the probability of influenza pandemic outbreak has been greatly decreased. However, influenza A virus (IAV), a negative-sense RNA virus with 8 separate segments in its genome [3], is very easy to mutate, which results in novel hemagglutinin (HA) production [4]. These mutations render IAV to invade easily population without immunity [5]. Studies have shown that it is the synergy between the viruses and bacteria that presents a great threat to public health while the viral infection alone rarely causes severe consequence [6-8]. Outcomes of influenza infection vary with age. Secondary bacterial infection has been considered as a key factor responsible for IAV death $[7,9]$. Among them, Streptococcus pneumoniae (SP) and Staphylococcus aureus (SA) are the most commonly seen bacterial types $[10,11]$. Additionally, the cytokine burst is another main cause of IAV-related death. Cytokine burst causes severe immunopathological damage to body [12] and also increases susceptibility to secondary bacterial infection [13].

The timing recognition of IAV and bacterial infection can be dated back to 1918 "Spanish flu." At that time, pneumoniaassociated deaths closely followed the outbreak of influenza. A set of bacteria such as SP and SA were incubated from these autopsies $[14,15]$. Clinical data have suggested that secondary bacterial infection usually occurs 1-2 weeks after IAV infection and frequently causes IAV-related death [16, 17]. In exploring the pathogenic mechanisms underlying the excess pneumonia mortality after influenza infections, various animals were used as in vivo models to investigate viral-bacterial interaction. Among them, a murine model established by McCullers et al. preferably manifested the clinical characterizations [18, 19]. In their study, IAV and SP were used to infect mice at various time sequence or pathway, which produced an intriguing mortality difference. The mortality was $60 \%$ in mice infected with IAV and SP simultaneously. It was up to $100 \%$ when SP was inoculated 7 days after IAV infection. On contrast, mice challenged with SP 7 days before IAV survived $100 \%$. Mice infected with either IAV or SP alone had mortalities of $35 \%$ or $15 \%$, 
respectively. Studies from other labs also supported that influenza infection followed by bacterial challenge rendered the most severe outcomes [20-24]. Thus, these results suggest IAV infection facilitates secondary SP infection.

(2) Prevailing Mechanisms of IAV Facilitating Hosts to Bacterial Pneumonia. Multiple factors are involved in virally bacterial pneumonia. Of them, respiratory epithelial damage is considered as the most classical one since the 1918 pandemic; that is, IAV incursion exposes the binding sites to bacteria $[19,25,26]$. This has been further proved by increasing number of pathological researches involving viral and bacterial infection [27-29]. In addition, the recognition of IAV by toll-like receptors (TLRs) increased interferons (IFNs) secretion. The latter suppressed the functions of macrophages and neutrophils and led to the failure of bacterial clearance $[30,31]$. To observe the dynamics of timing and sequential infection between IAV and SP, Shrestha et al. developed a mathematical model to simulate these processes [32]. Consistent with results of McCullers and Rehg that pneumococcal challenge 7 days after IAV infection leads to the most severe disease state and rapid death [19], Shrestha et al. found that SP infection 4-6 days after influenza infection was the most efficient model to cause invasive pneumonia [32]. One main cause for the delayed SP infection is believed to be due to the inhibition of alveolar macrophages by IFN$\gamma$, a product after IAV recognition by TLRs (Figure 1(a)) [33]. Other IAV-related factors promoting bacterial susceptibility include mucociliary dysfunction [34], bacterial receptors expressing on epithelial cells $[35,36]$, cytokines increasing vascular permeability (e.g., interleukin-16 (IL-6) and tumor necrosis factor- $\alpha$ (TNF- $\alpha)$ ) [37], cytokines inhibiting early immune response (e.g., IL-35, IL-17, and IL-10) [30, 38, 39]. Investigations involving classical innate and adaptive immune responses on IAV infection and its sequelae have been well documented (as reviewed in [25, 40-42]). However, the role of apoptosis and autophagy is less reported, especially in IAV-related bacterial pneumonia.

\section{Role of Autophagy and Apoptosis in Viral-Bacterial Interaction}

Interestingly, recent studies have shown that autophagy, an evolutionarily conserved cellular pathway existing ubiquitously in eukaryotes to degrade unwanted cytoplasmic materials such as long-lived proteins and organelles under stressed conditions like nutrition deprivation and hypoxia $[43,44]$ is involved in IAV infection $[45,46]$. Autophagy was initially found to protect against microbial invasion. Some viruses, such as influenza viruses, have evolved to subvert this mechanism for their own benefit [47]. Zhou et al. first reported that autophagy was related to IAV replication and that virus yield was decreased by autophagy suppression [45]. They also reported that viral titer was decreased by enhanced autophagy at another study [48]. Additionally, autophagy has been considered as a new programmed cell death way. Evidences showed that cell death induced by H5N1 is predominantly autophagic rather than apoptotic. Autophagic cell death was considered as a main factor causing severe lung injury in H5N1-infected mice. This injury can be ameliorated by suppressing autophagy but not apoptosis [49]. These data suggest that autophagy, to a certain extent, is involved in H5N1-related cell death both in vitro and in vivo. Nevertheless, in some pathologic situation, excessive autophagy might also lead to cell death through apoptosis (as reviewed in [50]). Although autophagic cell death was more likely to be induced in highly pathogenic strains $[49,51]$, whether autophagy is a way of executing cell death or cell death is accompanied by autophagy remain controversial $[50,52]$.

Apoptosis, classified as type I programmed cell death, is generally characterized by nuclear fragmentation, chromatin condensation, cell shrinkage, plasma membrane blebbing, and intact cell membrane [53-55]. Relationship between IAV and apoptosis was early studied in vitro and in vivo [5658]. Apoptosis was originally thought not to cause inflammation. Later studies showed that IAV induced caspase- 1 and caspase-3, which proteolytically processed IL- $1 \beta$ and IL-18, and subsequently indirectly caused inflammatory responses [59], including in respiratory epithelial cells and leucocytes [60]. By recognizing viral RNA, members of nucleotidebinding domain and leucine-rich-repeat-containing (NLRs) family such as cryopyrin assemble inflammasomes to activate caspase- 1 and then increase IL- $1 \beta$ and IL-18 secretion in macrophages $[61,62]$. Thus, it is stimuli inducing apoptosis that determine whether apoptosis causes inflammatory response [63].

Many IAV proteins are involved in apoptosis, such as nucleoprotein (NP), matrix protein 1 (M1), matrix protein 2 (M2), nonstructural protein (NS1) [64], and PB1-F2 [65]. Fourteen years ago, in investigating an unknown antigenic peptide of IAV presented by CD8+ T lymphocytes, Chen et al. found a protein, PB1-F2, the eleventh viral protein encoded by the open reading frame (ORF) of $\mathrm{PB} 1$ gene. This strain specific protein is considered as one of the virulence factors contributing to the high pathogenicity of IAV, including the ability in promoting secondary bacterial infection by inducing cytokine storm $[66,67]$. Thus, IAV tends to cause an inflammatory apoptosis.

\subsection{Autophagy and Apoptosis Facilitates Secondary Bacterial Pneumonia after IAV Infection}

2.1.1. IAV Induces Autophagy. Several IAV proteins are involved in progress of viral infection promoting bacterial superinfection via the regulation of autophagy and apoptosis, in which autophagy and apoptosis appear to be sequential events (Figure 1(a)). NS1 protein was expressed at the early stage of IAV infection [68]. It was reported that NS1 protein indirectly promoted autophagy at the early stage of IAV infection through upregulating the synthesis of $\mathrm{HA}$ and M2 [69] and downregulating apoptosis to facilitate viral replication [70]. NS1 protein also positively regulated PI3KAkt pathway to inhibit apoptosis in the early stage of IAV infection, while in the late stage, NS1 induced p53 dependent or independent pathways to activate apoptosis [71]. Besides, NS1 may suppress apoptosis partly by antagonizing IFN. 


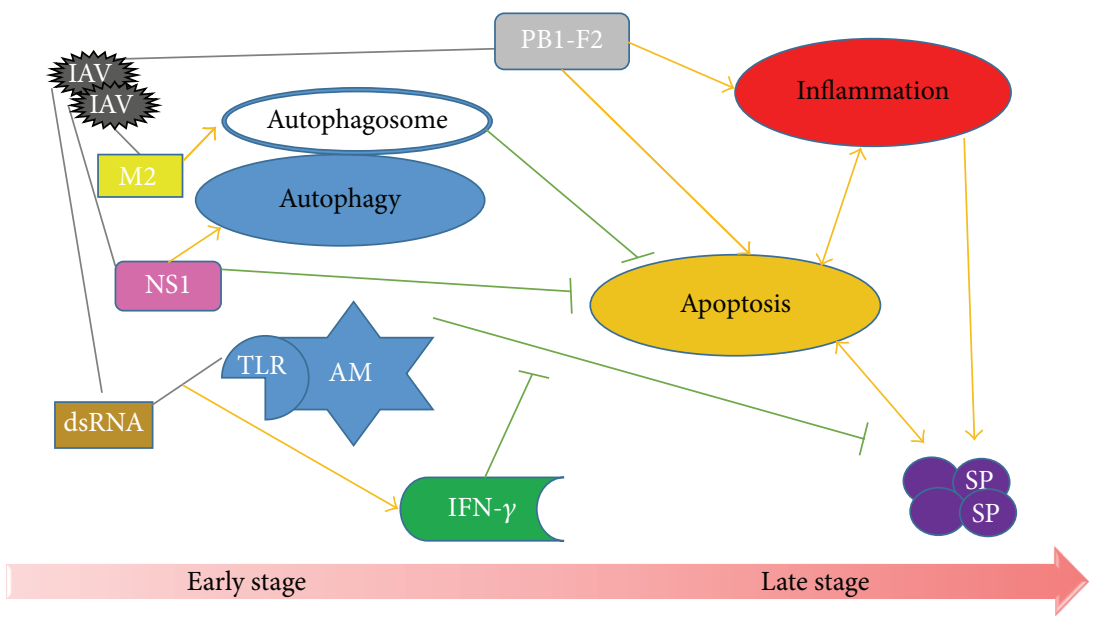

(a)

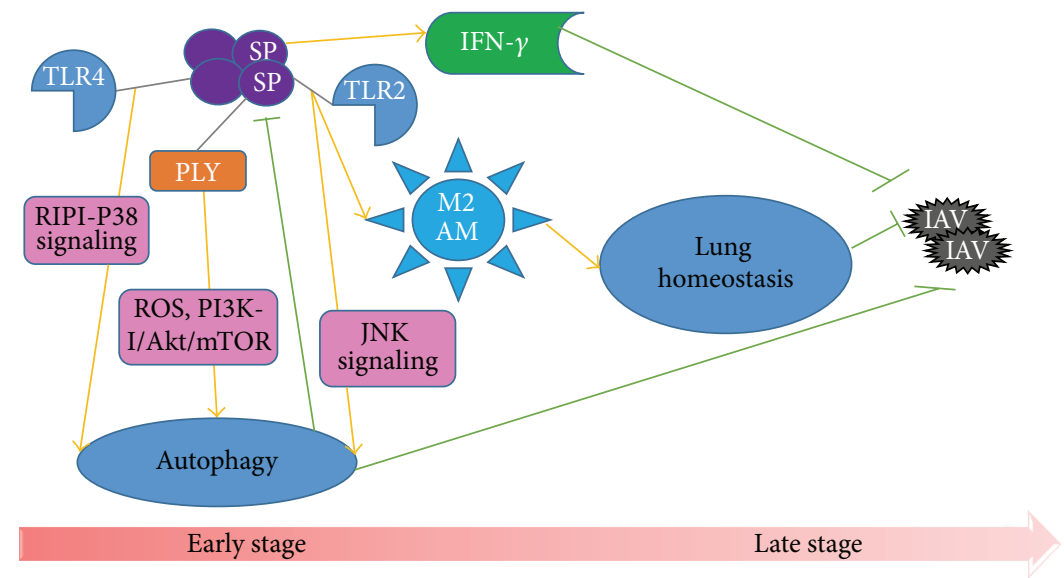

(b)

FIGURE 1: Autophagy and apoptosis in the early and late stage of IAV-SP mixed infection. In the figure, the orange arrows represent links of stimulation, whereas the green bars correspond to inhibitory links. (a) Autophagy and apoptosis seem to act as sequential events after IAV infection. NS1 plays a critical role in regulating IAV-induced autophagy and apoptosis. M2 is necessary for the formation of autophagosomes. The latter delays the development of apoptosis. PB1-F2 induces inflammatory response, which has a mutual promotion with apoptosis. PB1-F2 of PR8 can cause apoptosis of monocytes. Both apoptosis and inflammation contribute to secondary bacterial infection. Recognition of viral dsRNA by TLR of alveolar macrophages promotes IFN- $\gamma$ production, which prevents macrophages from clearing bacteria (such as SP). (b) SP triggers autophagy by interacting with TLR4 and TLR2 through RIPI-P38 signaling and JNK signaling, respectively. By the interaction of SP and TLR2, M2 alveolar macrophages (M2 AM) polarized from monocytes help maintain the lung homeostasis. PLY stimulate autophagy through ROS hypergeneration and PI3K-I/Akt/mTOR pathway. In addition, SP causes IFN- $\gamma$ increase, together with autophagy and the maintenance of lung homeostasis, which alleviates subsequent IAV infection.

Apoptosis was enhanced and accelerated in IFN-competent cells infected by NS1-depeleted variant (delNS1) but was delayed in IFN-deficient cells infected by wild-type or delNS1 strains [70]. Furthermore, NS1 also exerted antiapoptosis effect by blocking the recognition of recognizing doublestranded RNA (dsRNA) dependent protein kinase (PKR) to viral dsRNA [72-75]. Notably, apoptosis suppressive effect of NS1 is strain specific. NS1 exhibited apoptosis suppression in some strains, like H1N1, H3N2, and $\mathrm{H} 7 \mathrm{~N} 7$ [70, 76]. Conversely, in some strains, like H5N9 and H5N1, NS1 promoted apoptosis $[77,78]$. M2 protein acted as a proton channel for viral uncoating after fusion in endosomes [3]. M2 protein is also found to be necessary for autophagosome formation. Silencing M2 expression resulted in significantly reduced autophagosome accumulation during IAV infection. However, M2 blocked IAV-induced fusion of autophagosome and lysosome via binding to Beclin-1 with its first 60 amino acids [79]. As autophagy deficient cells exhibited enhanced apoptosis, M2-mediated autophagosome accumulation is likely to decrease apoptosis [79]. On the whole, influenza virus provides environment and time for viral replication by inhibiting apoptosis and triggering autophagy at the early stage of infection.

2.1.2. Autophagy in IAV Infection: Two Sides of a Coin. Autophagy is a doubled edge sword. On the one hand, 
autophagy is a prosurvival pathway in which it protects against various pathogenic invasions, including IAV [80]. The antiviral character of autophagy is mostly attributed to its function in adaptive immunity. One recent study showed that autophagy was essential for the maintenance of memory B cells against IAV infection in vivo [81]. In this study, Chen et al. demonstrated that mice with autophagyrelated gene (Atg) 7 knockdown in B cells failed to produce secondary antibodies when they were infected with IAV again [81]. Similarly, autophagy displays a prosurvival role in effector CD8+ T cells during influenza infection. By using an inducible Atg5 knockout mouse system, Schlie et al. found that mice infected with IAV failed to recall a primary response peak, and the Atg5-/- CD8+ T cells exhibited feeble viability and upregulated P53 expression [82]. Additionally, a natural compound, pentagalloylglucose (PGG), which was reported to have anti-influenza activity [83], promoted autophagic flux via degradation of viral M2 protein, a protein that blocks the fusion of autophagosome and lysosome at enough high concentration, and subsequently caused the downregulation of several viral proteins, like NP, M1, HA, and M2 [84]. On the other hand, autophagy is beneficial to IAV replication and production. Zhou et al. reported in an in vitro experiment that autophagy was involved in IAV replication and autophagy suppression and decreased viral yield [45]. A large amount of studies showed that autophagic deficiency reduced IAV virulence $[45,51,84-91]$. It is noteworthy that IAVinduced autophagy is both strain and cell specific $[46,92]$. In addition, autophagy is associated with influenza-induced inflammatory. For instance, TLR3 enhanced autophagy by dsRNA to promote the production of IFN and some other cytokines $[93,94]$. Autophagy was also involved in the induction of IFN- $\alpha$ and CXCL10 in H9N2/G1 infected cells [46, 92]. Moreover, autophagy-mediated inflammatory response was also associated with nuclear factor- $\kappa \mathrm{B}(\mathrm{NF}-\kappa \mathrm{B})$ and p38 mitogen-activated protein kinase (MAPK) signaling in $\mathrm{H} 5 \mathrm{~N} 1$ pseudovirus infection. This signaling in turn promoted the formation of autophagosomes, suggesting an important mechanism underlying H5N1-related hypercytokemia [95].

2.1.3. IAV-Induced Apoptosis: More of a Foe Than a Friend. Apoptosis has dual characters as well as autophagy. Several proapoptotic factors definitely play an antiviral role. Recently, Chang et al. found that several avian influenza viruses induced early apoptosis in porcine alveolar macrophages, which inhibited viral replication and mitigated inflammation [96]. IL-24 was found to decrease IAV titer by activating TLR3 dependent apoptosis [97]. Although initial findings show that apoptosis is a host defensive mechanism against IAV infection [98], generally speaking, apoptosis is beneficial to viral replication, dissemination, and host immune cells kill. As such, apoptosis may serve as a contributor for secondary bacterial infection following influenza virus infection.

Firstly, IAV indeed triggers apoptosis through various mechanisms to damage host immunity ability. Some viral components, such as NS1, M2, PB1-F2, M1, NA (neuraminidase), NP, and dsRNA, are associated with apoptosis regulation. As described above, viral NS1 plays an antiapoptotic role in host immune response [69-75]. Combined with M2, these viral components regulate autophagy and apoptosis as sequential events [79]. PB1-F2, a viral protein encoded by an open reading frame of IAV PB1, is shown to induce apoptosis at the late stage of IAV infection via mitochondrial permeabilization in strain dependent and cell specific manners [65]. It is notable that only PB1-F2 produced by influenza A/Puerto Rico/8/34 (H1N1) (hereafter referred as to PR8), but not other strains, induced alveolar macrophages death rather than epithelial cells in the lung $[65,99,100]$. As a result, PB1-F2 interferes in viral and bacterial clearance and antigen presentation at the early stage. McAuley et al. introduced PB1-F2 protein of 1918 influenza H1N1 virus into PR8, resulting in a higher susceptibility to secondary bacterial pneumonia than wild-type PR8. Nonetheless, they also observed that PB1-F2 knockout variant resulted in lower mortality when followed by SP infection as compared to wildtype PR8, which expresses PB1-F2 with 87 amino acids [101], although both had similar viral loads in lung [66]. Recently, Yoshizumi et al. found that full-length PB1-F2 of highly pathogenic IAVs translocated into mitochondria via Tom 40 channels and then impaired innate immune and contributed to symptomatic deterioration, while truncated PB1-F2 (lacking C-terminal region responsible for translocating into mitochondria) from low pathogenic IAVs was less harmful due to disability in translocating into mitochondria [102]. These findings indicate that PB1-F2 exerts the pathogenicity on postinfluenza bacterial infection more likely through other mechanisms rather than apoptosis. One of the most direct causes is excessive inflammation [103-105]. An in vivo experiment with different viral strains discovered that PB1F2 was related to inflammatory infiltration of macrophages and neutrophils, hypertrophy of epithelial cells, and fibrin deposition [67]. Additionally, PB1-F2 induced inflammatory response by activating inflammasome [106], regulating NF- $\kappa \mathrm{B}$ and IKK $\beta$ activity [107] and forming aggregates [108]. $\mathrm{M} 1$ and NA protein induced apoptosis by interacting with caspase-8 $[109,110]$ or activating tumor growth factor- $\beta$ (TGF- $\beta$ ) [77, 111]. Human Clusterin (CLU) prevented intrinsic apoptosis pathway through binding to Bax, which interfered with viral NP protein [112]. dsRNA virus-mediated apoptosis was reported to be related to caspase-dependent pathway [113], PKR, TLR, retinoic acid-inducible gene (RIG), and other forms of signaling [114-117].

Secondly, many reports have shown that apoptosis inhibition decreases IAV pathogenicity. Herold et al. reported that macrophages, when recruited from peripheral blood to the lungs during IAV infection, released tumor necrosis factor-related apoptosis-inducing ligand (TRAIL) to induce apoptosis of alveolar epithelial cells and increase lung leakage and mortality, which in turn were rescued by blocking TRAIL signaling [118]. Additionally, Liu et al. found that caspase inhibitors decreased viral replication and release of certain kinds of proinflammatory cytokines and chemokines in IAV infected mast cells [119]. Jaworska et al. discovered that the interaction of host NLRX1 and viral PB1-F2 protein suppressed mitochondria-related apoptosis and enhanced macrophage function, which, as a result, mitigated viral replication, lung function disorder, and mortality [120]. Tran et al. 
used human whole-genome screen method to search for cell death related genes in IAV infection. USP47, TNF superfamily (TNFSF) 13, and TNFSF12-13 were identified as important components. Their depletion produced host protective effects [121].

As mentioned above, SP significantly aggravates IAV infection. To explore the role of apoptosis in postinfluenza SP pneumonia, Kosai et al. infected mice with IAV or SP alone or IAV 48 hours followed by SP. They found that apoptosis occurred earlier and more severe in mice infected with combination of IAV and SP than IAV or SP alone [122].

\subsection{Preceding SP Infection Alleviates Onsets of Subsequent IAV Invasion: Role of Autophagy}

2.2.1. Preceding Bacterial Infection Protects Host from IAV Infection. A prior bacterial exposure may protect the host from adverse impacts of following IAV infection. This concern is mainly derived from study made by McCullers and Rehg. In their study, mice infected with IAV 7 days after SP had $0 \%$ mortality, while other groups suffered from mortality from $25 \%$ to $100 \%$ [19]. Deprivation of commensal bacteria (such as SP and SA) from respiratory tract exacerbated influenza-induced disorder [123-125]. Recently, an in vivo experiment showed that preceding SP infection protected mice from IAV-related detriment [126]. One of possible mechanisms underlying this virus antagonistic effect is that bacteria create an inflammatory environment [40]. Indeed, SP infection promoted IFN- $\gamma$ production [127-131], which results in an antiviral state (Figure $1(\mathrm{~b})$ ). However, the role of autophagy and apoptosis in this phenomenon is paid less attention.

2.2.2. Role of Autophagy in Postbacterial IAV Infection. Autophagy seems to play a critical role in improving host immunity in SP infection (Figure 1(b)). Guo et al. found that SP-induced autophagy was a defense mechanism against bacterial infection [132]. The mechanism may be partly attributed to recognition of LPS of SP by TLR4 to trigger autophagy through receptor-interacting protein 1 (RIP1-P38) signaling to promote SP clearance $[133,134]$. Also, Li et al. reported that SP clearance was enhanced by autophagy; conversely bacterial clearance was reduced by autophagy suppression [135]. In addition, although preadministrated SP failed to produce any detectable effects on either cell morphology or IAV replication in epithelial cells [136], another in vivo study conducted by Wang et al. showed bacteria colonization, to some extent, indeed preventing viral infection [125]. Comparing to SA-free mice and wild-type mice, specific pathogenfree (SPF) mice were more susceptible to fatalness induced by IAV [125]. This is consistent with the results from McCullers and Rehg, although different cocci were used [19]. One of possible mechanisms underlying this result is that peripheral CCR2+CD11b+ monocytes were recruited into alveoli and then were polarized to M2 alveolar macrophages by the interaction between SA and TLR2. Therefore, SA colonization increases immunity ability. Nonetheless, as TLR2 is crucial for lung homeostasis rather than bacterial elimination,
TLR2 deficiency failed to interfere in SA elimination [125]. TLR2 was also reported to trigger autophagy through JNK signaling [137]. In this situation, TLR2 could serve as a stimulus for the development of bacteria-induced autophagy. These may explain why Ouyang et al. did not detect the influence of SP pretreatment on IAV replication. Recently, Wolf et al. reported that pneumolysin, a bacterial virulence factor important in inducing immune responses, protected postpneumococcus IAV infection in an in vivo model [126]. Li et al. further supported that pneumolysin was a key factor in triggering autophagy through ROS hypergeneration and inhibition of PI3K-I/Akt/mTOR pathways in A549 cells [135]. In other words, SP might exert protective effects against IAV via autophagy mechanism. Taken together, it is clear that preceding SP infection produces systematic defense reactions, including autophagy to attenuate the followed IAV infection.

\section{Role of Autophagy and Apoptosis in Viral-Bacterial Coinfection: A Potential Research Field}

3.1. Present Treatment against IAV and Bacterial Infection. Present treatments against IAV and bacterial infection include viral and bacterial vaccines, antiflu drugs, and antibiotics. As reviewed by Christopoulou et al., application of viral and bacterial vaccines effectively decreases post-IAV bacterial infection [138]. Antiflu drugs are also shown to not only reduce IAV infection but also decrease clinical morbidity of secondary bacterial infection $[139,140]$. Nonetheless, as IAV is an ssRNA virus with 8 segments, which contribute to low self-correcting ability during transcription, IAVs are easily to produce new mutants which are consequently resistant to antiviral drugs or fail to be neutralized by vaccines [42, 141, 142]. Additionally, multidrug resistant SA (MDRSA), especially methicillin-resistant SA (MRSA), have been widely disseminated in hospital and community $[143,144]$. For these concerns, although vaccines and antibiotics are currently primary treatments for possible post-IAV bacterial infections, further mechanism exploration for more treatment targets, including autophagy and apoptosis, is still significantly important.

3.2. Autophagy and Apoptosis Regulation: A Potential Alternative Method to Fight against Increased Susceptibility to Post$I A V$ Bacterial Infection. Here, we propose an assumption that regulating autophagy at the early stage or suppressing apoptosis at the late stage may be a promising strategy to antagonize post-IAV SP pneumonia. Until now, there are a number of studies showing that autophagy suppression ameliorates the impact of IAV risk [45, 51, 84-91]. Although IAV has been proved to inhibit autophagy to facilitate its replication, decreased viral titer was also observed in presence of autophagy stimulator rapamycin in MDCK cells [48]. What is more, autophagy plays a protective role in adaptive immunity against virus-infected hosts [81, 82]. Some chemicals exert virus suppressive effect by triggering autophagy [83, 84]. These indicate that excessive or insufficient autophagy is detrimental for IAV. 
What needs to be paid special attention is a moderate regulation of autophagy. Hahn et al. established a mouse model in which Atg5 gene was knockout in the distal respiratory epithelium to achieve different degrees of autophagic reduction [145]. They infected these mice with $50 \%$ autophagy ability with $\mathrm{H} 3 \mathrm{~N} 2$ virus. The results showed that viral replication was decreased, lung structure and function were improved, and morbidity and mortality were decreased. When mice with 10\% autophagy ability were used, lung injury in elderly group was exacerbated with time, while alveolar septum was thickened in adult group. Therefore, an appropriate autophagic level is necessary to fight against IAV invasion. As aforementioned, inhibiting apoptosis presents an overall beneficial effect for hosts infected by IAV [118121]. Thus, treating IAV infection with autophagy regulators and apoptosis inhibitors in in vivo models can be regarded as a potential researching field to explore a new breakthrough fighting against influenza and its sequelae.

The significance of this assumption is that the high conservativeness of autophagy and apoptosis in eukaryotes, to some extent, prevents the complexity of IAV mutation. The concept that autophagy and apoptosis are conserved among eukaryotes has been applied in drug screening and discovery, where cell-based assays were used for antiviral drug screening $[86,87,89]$. Further validation is necessary in in vivo models.

\section{Conclusion}

Collectively, IAV facilitates its host to suffer from bacterial pneumonia via various pathways. Among the underlying mechanisms, autophagy and apoptosis act as sequential events to regulate post-IAV bacterial pneumonia. Systemic analysis of autophagy and apoptosis may provide a new strategy for prophylactic and therapeutic treatment of influenza virus infection.

\section{Competing Interests}

The authors declared no competing interests.

\section{Acknowledgments}

This work was financially supported by the National Science Foundation of China (Grant no. 30671964) and State Key Laboratory of Oral Diseases (Grant no. SKLOD2015OF08).

\section{References}

[1] Y.-M. Loo and M. Gale Jr., "Influenza: fatal immunity and the 1918 virus," Nature, vol. 445, no. 7125, pp. 267-268, 2007.

[2] N. P. A. S. Johnson and J. Mueller, "Updating the accounts: global mortality of the 1918-1920 'Spanish' influenza pandemic," Bulletin of the History of Medicine, vol. 76, no. 1, pp. 105-115, 2002.

[3] P. Palese and M. Shaw, "Orthomyxoviridae: the viruses and their replication," in Fields Virology, D. M. Knipe and P. M. Howley, Eds., pp. 1647-1689, 4th edition, 2007.
[4] K. S. Li, Y. Guan, J. Wang et al., "Genesis of a highly pathogenic and potentially pandemic $\mathrm{H} 5 \mathrm{~N} 1$ influenza virus in eastern Asia," Nature, vol. 430, no. 6996, pp. 209-213, 2004.

[5] P. Palese, "Influenza: old and new threats," Nature Medicine, vol. 10, no. 12, pp. S82-S87, 2004.

[6] K. L. O’Brien, M. I. Walters, J. Sellman et al., "Severe pneumococcal pneumonia in previously healthy children: the role of preceding influenza infection," Clinical Infectious Diseases, vol. 30, no. 5, pp. 784-789, 2000.

[7] D. M. Morens, J. K. Taubenberger, and A. S. Fauci, "Predominant role of bacterial pneumonia as a cause of death in pandemic influenza: implications for pandemic influenza preparedness," Journal of Infectious Diseases, vol. 198, no. 7, pp. 962-970, 2008.

[8] J. Muscedere, M. Ofner, A. Kumar et al., "The occurrence and impact of bacterial organisms complicating critical care illness associated with 2009 influenza A(H1N1) infection," Chest, vol. 144, no. 1, pp. 39-47, 2013.

[9] L. Simonsen, "The global impact of influenza on morbidity and mortality," Vaccine, vol. 17, no. 1, pp. S3-S10, 1999.

[10] K. P. Klugman, Y.-W. Chien, and S. A. Madhi, "Pneumococcal pneumonia and influenza: a deadly combination," Vaccine, vol. 27, no. 3, pp. C9-C14, 2009.

[11] S. A. Madhi, K. P. Klugman, and Vaccine Trialist Group, "A role for Streptococcus pneumoniae in virus-associated pneumonia," Nature Medicine, vol. 10, no. 8, pp. 811-813, 2004.

[12] C. C. J. Zavitz, C. M. T. Bauer, G. J. Gaschler et al., "Dysregulated macrophage-inflammatory protein-2 expression drives illness in bacterial superinfection of influenza," Journal of Immunology, vol. 184, no. 4, pp. 2001-2013, 2010.

[13] E. J. Mifsud, A. C. Tan, K. R. Short, L. E. Brown, B. Y. Chua, and D. C. Jackson, "Reducing the impact of influenzaassociated secondary pneumococcal infections," Immunology and Cell Biology, vol. 94, pp. 101-108, 2016.

[14] G. A. Soper, "The pandemic in the Army camps," Journal of the American Medical Association, vol. 71, pp. 1899-1909, 1918.

[15] L. H. Spooner, J. M. Scott, and E. H. Heath, "A bacteriologic study of the influenza epidemic at Camp Devens," Journal of the American Medical Association, vol. 72, no. 3, pp. 155-159, 1919.

[16] Prevention CDC, "Influenza," CDC [homepage on the Internet], 2012, April 2016, http://www.cdc.gov/vaccines/pubs/pinkbook/flu.html.

[17] J. F. Brundage, "Interactions between influenza and bacterial respiratory pathogens: implications for pandemic preparedness," Lancet Infectious Diseases, vol. 6, no. 5, pp. 303-312, 2006.

[18] J. A. McCullers and R. G. Webster, "A mouse model of dual infection with influenza virus and Streptococcus pneumoniae," International Congress Series, vol. 1219, pp. 601-607, 2001.

[19] J. A. McCullers and J. E. Rehg, "Lethal synergism between influenza virus and Streptococcus pneumoniae: characterization of a mouse model and the role of platelet-activating factor receptor," Journal of Infectious Diseases, vol. 186, no. 3, pp. 341350, 2002.

[20] M. Seki, Y. Higashiyama, K. Tomono et al., "Acute infection with influenza virus enhances susceptibility to fatal pneumonia following Streptococcus pneumoniae infection in mice with chronic pulmonary colonization with Pseudomonas aeruginosa," Clinical and Experimental Immunology, vol. 137, no. 1, pp. 35-40, 2004.

[21] J. A. McCullers, J. L. McAuley, S. Browall, A. R. Iverson, K. L. Boyd, and B. H. Normark, "Influenza enhances susceptibility 
to natural acquisition of and disease due to Streptococcus pneumoniae in ferrets," Journal of Infectious Diseases, vol. 202, no. 8, pp. 1287-1295, 2010.

[22] L. A. McNamee and A. G. Harmsen, "Both influenza-induced neutrophil dysfunction and neutrophil-independent mechanisms contribute to increased susceptibility to a secondary Streptococcus pneumoniae infection," Infection and Immunity, vol. 74, no. 12, pp. 6707-6721, 2006.

[23] G. T. Ellis, S. Davidson, S. Crotta, N. Branzk, V. Papayannopoulos, and A. Wack, "TRAIL ${ }^{+}$monocytes and monocyte-related cells cause lung damage and thereby increase susceptibility to influenza-Streptococcus pneumoniae coinfection," EMBO Reports, vol. 16, no. 9, pp. 1203-1218, 2015.

[24] J. Berg, K. Hellwig, D. Stoll et al., "IVA induced IFNs facilitate development of secondary pneumococcal pneumonia in human lung tissue," Pneumologie, vol. 69-A1, 2015.

[25] J. A. McCullers, "Insights into the interaction between influenza virus and pneumococcus," Clinical Microbiology Reviews, vol. 19, no. 3, pp. 571-582, 2006.

[26] M.-C. Plotkowski, E. Puchelle, G. Beck, J. Jacquot, and C. Hannoun, "Adherence of type I Streptococcus pneumoniae to tracheal epithelium of mice infected with influenza A/PR8 virus," American Review of Respiratory Disease, vol. 134, no. 5, pp. 1040-1044, 1986.

[27] T. Mauad, L. A. Hajjar, G. D. Callegari et al., "Lung pathology in fatal novel human influenza A (H1N1) infection," American Journal of Respiratory and Critical Care Medicine, vol. 181, no. 1, pp. 72-79, 2010.

[28] C. L. Nickerson and G. J. Jakab, "Pulmonary antibacterial defenses during mild and severe influenza virus infection," Infection and Immunity, vol. 58, no. 9, pp. 2809-2814, 1990.

[29] J. Louie, C. Jean, T. H. Chen et al., "Bacterial coinfections in lung tissue specimens from fatal cases of 2009 pandemic influenza A (H1N1)-United States, May-August 2009," Morbidity and Mortality Weekly Report, vol. 58, no. 38, pp. 1071-1074, 2009.

[30] W. Li, B. Moltedo, and T. M. Moran, "Type I interferon induction during influenza virus infection increases susceptibility to secondary Streptococcus pneumoniae infection by negative regulation of $\gamma \delta$ T cells," Journal of Virology, vol. 86, no. 22, pp. 12304-12312, 2012.

[31] B. Lee, K. M. Robinson, K. J. McHugh et al., "Influenza-induced type I interferon enhances susceptibility to gram-negative and gram-positive bacterial pneumonia in mice," American Journal of Physiology - Lung Cellular and Molecular Physiology, vol. 309, no. 2, pp. L158-L167, 2015.

[32] S. Shrestha, B. Foxman, S. Dawid et al., "Time and dose-dependent risk of pneumococcal pneumonia following influenza: a model for within-host interaction between influenza and Streptococcus pneumoniae," Journal of the Royal Society Interface, vol. 10, no. 86, article 0233, 2013.

[33] K. Sun and D. W. Metzger, "Inhibition of pulmonary antibacterial defense by interferon- $\gamma$ during recovery from influenza infection," Nature Medicine, vol. 14, no. 5, pp. 558-564, 2008.

[34] L. A. Pittet, L. Hall-Stoodley, M. R. Rutkowski, and A. G. Harmsen, "Influenza virus infection decreases tracheal mucociliary velocity and clearance of Streptococcus pneumoniae," American Journal of Respiratory Cell and Molecular Biology, vol. 42, no. 4, pp. 450-460, 2010.

[35] V. Avadhanula, C. A. Rodriguez, J. P. De Vincenzo et al., "Respiratory viruses augment the adhesion of bacterial pathogens to respiratory epithelium in a viral species- and cell typedependent manner," Journal of Virology, vol. 80, no. 4, pp. 16291636, 2006.

[36] N. Li, A. Ren, X. Wang et al., "Influenza viral neuraminidase primes bacterial coinfection through TGF- $\beta$-Mediated expression of host cell receptors," Proceedings of the National Academy of Sciences of the United States of America, vol. 112, no. 1, pp. 238-243, 2015.

[37] S. Wang, T. Q. Le, N. Kurihara et al., "Influenza virus-cytokineprotease cycle in the pathogenesis of vascular hyperpermeability in severe influenza," Journal of Infectious Diseases, vol. 202, no. 7, pp. 991-1001, 2010.

[38] Y. Chen, C. J. Wang, S. H. Lin, M. Zhang, S. Y. Li, and F. Xu, "Interleukin-35 is upregulated in response to influenza virus infection and secondary bacterial pneumonia," Cytokine, vol. 81, pp. 23-27, 2016.

[39] K. F. van der Sluijs, L. J. R. Van Elden, M. Nijhuis et al., "IL10 is an important mediator of the enhanced susceptibility to pneumococcal pneumonia after influenza infection," The Journal of Immunology, vol. 172, no. 12, pp. 7603-7609, 2004.

[40] J. A. McCullers, "The co-pathogenesis of influenza viruses with bacteria in the lung," Nature Reviews Microbiology, vol. 12, no. 4, pp. 252-262, 2014.

[41] K. R. Short, M. N. Habets, P. W. M. Hermans, and D. A. Diavatopoulos, "Interactions between Streptococcus pneumoniae and influenza virus: a mutually beneficial relationship?" Future Microbiology, vol. 7, no. 5, pp. 609-624, 2012.

[42] A. M. Smith and J. A. McCullers, "Secondary bacterial infections in influenza virus infection pathogenesis," Current Topics in Microbiology and Immunology, vol. 385, pp. 327-356, 2014.

[43] Z. Yang and D. J. Klionsky, "Eaten alive: a history of macroautophagy," Nature Cell Biology, vol. 12, no. 9, pp. 814-822, 2010.

[44] D. J. Klionsky and S. D. Emr, "Autophagy as a regulated pathway of cellular degradation," Science, vol. 290, no. 5497, pp. 1717-1721, 2000.

[45] Z. Zhou, X. Jiang, D. Liu et al., "Autophagy is involved in influenza A virus replication," Autophagy, vol. 5, no. 3, pp. 321328, 2009.

[46] A. H. Y. Law, D. C. W. Lee, and A. S. Y. Lau, "Role for autophagy in cellular response to influenza virus infection," Hong Kong Medical Journal, vol. 20, supplement 6, pp. S20-S24, 2014.

[47] B. Yordy and A. Iwasaki, "Autophagy in the control and pathogenesis of viral infection," Current Opinion in Virology, vol. 1, no. 3, pp. 196-203, 2011.

[48] Z. Zhou, Association of influenza a virus with autophagy [Ph.D. dissertation], National Digital Library of China, 2007 (Chinese).

[49] Y. Sun, C. Li, Y. Shu et al., "Inhibition of autophagy ameliorates acute lung injury caused by avian influenza A H5N1 infection," Science Signaling, vol. 5, no. 212, article ra16, 2012.

[50] G. Kroemer and B. Levine, "Autophagic cell death: the story of a misnomer," Nature Reviews Molecular Cell Biology, vol. 9, no. 12, pp. 1004-1010, 2008.

[51] J. Ma, Q. Sun, R. Mi, and H. Zhang, "Avian influenza A virus H5N1 causes autophagy-mediated cell death through suppression of mTOR signaling," Journal of Genetics and Genomics, vol. 38, no. 11, pp. 533-537, 2011.

[52] J. Debnath, E. H. Baehrecke, and G. Kroemer, "Does autophagy contribute to cell death?” Autophagy, vol. 1, no. 2, pp. 66-74, 2005.

[53] S. Elmore, "Apoptosis: a review of programmed cell death," Toxicologic Pathology, vol. 35, no. 4, pp. 495-516, 2007. 
[54] R. A. Schwartzman and J. A. Cidlowski, "Apoptosis: the biochemistry and molecular biology of programmed cell death," Endocrine Reviews, vol. 14, no. 2, pp. 133-151, 1993.

[55] J. F. Kerr, A. H. Wyllie, and A. R. Currie, "Apoptosis: a basic biological phenomenon with wide-ranging implications in tissue kinetics," British Journal of Cancer, vol. 26, no. 4, pp. 239-257, 1972.

[56] T. Takizawa, S. Matsukawa, Y. Higuchi, S. Nakamura, Y. Nakanishi, and R. Fukuda, "Induction of programmed cell death (apoptosis) by influenza virus infection in tissue culture cells," Journal of General Virology, vol. 74, no. 11, pp. 2347-2355, 1993.

[57] V. S. Hinshaw, C. W. Olsen, N. Dybdahl-Sissoko, and D. Evans, "Apoptosis: a mechanism of cell killing by influenza A and B viruses," Journal of Virology, vol. 68, no. 6, pp. 3667-3673, 1994.

[58] I. Mori, T. Komatsu, K. Takeuchi, K. Nakakuki, M. Sudo, and Y. Kimura, "In vivo induction of apoptosis by influenza virus," Journal of General Virology, vol. 76, no. 11, pp. 2869-2873, 1995.

[59] E. W. A. Brydon, S. J. Morris, and C. Sweet, "Role of apoptosis and cytokines in influenza virus morbidity," FEMS Microbiology Reviews, vol. 29, no. 4, pp. 837-850, 2005.

[60] I. Julkunen, K. Melén, M. Nyqvist, J. Pirhonen, T. Sareneva, and S. Matikainen, "Inflammatory responses in influenza A virus infection," Vaccine, vol. 19, no. 1, pp. S32-S37, 2000.

[61] I. C. Allen, M. A. Scull, C. B. Moore et al., "The NLRP3 inflammasome mediates in vivo innate immunity to influenza A virus through recognition of viral RNA," Immunity, vol. 30, no. 4, pp. 556-565, 2009.

[62] P. G. Thomas, P. Dash, J. R. Aldridge Jr. et al., "The intracellular sensor NLRP3 mediates key innate and healing responses to influenza A virus via the regulation of caspase-1," Immunity, vol. 30, no. 4, pp. 566-575, 2009.

[63] N. P. Restifo, "Building better vaccines: how apoptotic cell death can induce inflammation and activate innate and adaptive immunity," Current Opinion in Immunology, vol. 12, no. 5, pp. 597-603, 2000.

[64] J.-M. Hament, J. L. L. Kimpen, A. Fleer, and T. F. W. Wolfs, "Respiratory viral infection predisposing for bacterial disease: a concise review," FEMS Immunology and Medical Microbiology, vol. 26, no. 3-4, pp. 189-195, 1999.

[65] W. Chen, P. A. Calvo, D. Malide et al., "A novel influenza A virus mitochondrial protein that induces cell death," Nature Medicine, vol. 7, no. 12, pp. 1306-1312, 2001.

[66] J. L. McAuley, F. Hornung, K. L. Boyd et al., "Expression of the 1918 influenza A virus PB1-F2 enhances the pathogenesis of viral and secondary bacterial pneumonia," Cell Host and Microbe, vol. 2, no. 4, pp. 240-249, 2007.

[67] J. L. McAuley, J. E. Chipuk, K. L. Boyd, N. Van De Velde, D. R. Green, and A. M. Jonathan, "PB1-F2 proteins from $\mathrm{H} 5 \mathrm{~N} 1$ and 20th century pandemic influenza viruses cause immunopathology," PLoS Pathogens, vol. 6, no. 7, Article ID e1001014, pp. 1-12, 2010.

[68] R. Krug, Unique Functions of the NS1 Protein, Textbook of Inffuenza Blackwell Science, Oxford, UK, 1998.

[69] O. P. Zhirnov and H. D. Klenk, "Influenza a virus proteins NS1 and hemagglutinin along with M2 are involved in stimulation of autophagy in infected cells," Journal of Virology, vol. 87, no. 24, pp. 13107-13114, 2013.

[70] O. P. Zhirnov, T. E. Konakova, T. Wolff, and H.-D. Klenk, "NS1 protein of influenza A virus down-regulates apoptosis," Journal of Virology, vol. 76, no. 4, pp. 1617-1625, 2002.
[71] O. P. Zhirnov and H.-D. Klenk, "Control of apoptosis in influenza virus-infected cells by up-regulation of Akt and p53 signaling," Apoptosis, vol. 12, no. 8, pp. 1419-1432, 2007.

[72] E. Hatada, S. Saito, and R. Fukuda, "Mutant influenza viruses with a defective NS1 protein cannot block the activation of PKR in infected cells," Journal of Virology, vol. 73, no. 3, pp. 24252433, 1999.

[73] Y. Lu, M. Wambach, M. G. Katze, and R. M. Krug, "Binding of the influenza virus NS1 protein to double-stranded RNA inhibits the activation of the protein kinase that phosphorylates the eIF-2 translation initiation factor," Virology, vol. 214, no. 1, pp. 222-228, 1995.

[74] S.-L. Tan and M. G. Katze, "Biochemical and genetic evidence for complex formation between the influenza a virus NS1 protein and the interferon-induced PKR protein kinase," Journal of Interferon \& Cytokine Research, vol. 18, no. 9, pp. 757-766, 1998.

[75] S. Ludwig, X. Wang, C. Ehrhardt et al., "The influenza A virus NS1 protein inhibits activation of Jun N-terminal kinase and AP-1 transcription factors," Journal of Virology, vol. 76, no. 21, pp. 11166-11171, 2002.

[76] S. J. Morris, K. Nightingale, H. Smith, and C. Sweet, "Influenza A virus-induced apoptosis is a multifactorial process: exploiting reverse genetics to elucidate the role of influenza A virus proteins in virus-induced apoptosis," Virology, vol. 335, no. 2, pp. 198-211, 2005.

[77] S. Schultz-Cherry and V. S. Hinshaw, "Influenza virus neuraminidase activates latent transforming growth factor beta," Journal of Virology, vol. 70, no. 12, pp. 8624-8629, 1996.

[78] T. Daidoji, T. Koma, A. Du et al., "H5N1 avian influenza virus induces apoptotic cell death in mammalian airway epithelial cells," Journal of Virology, vol. 82, no. 22, pp. 11294-11307, 2008.

[79] M. Gannagé, D. Dormann, R. Albrecht et al., "Matrix protein 2 of influenza A virus blocks autophagosome fusion with lysosomes," Cell Host and Microbe, vol. 6, no. 4, pp. 367-380, 2009.

[80] T. Shintani and D. J. Klionsky, "Autophagy in health and disease: a double-edged sword," Science, vol. 306, no. 5698, pp. 990-995, 2004.

[81] M. Chen, M. J. Hong, H. Sun et al., "Essential role for autophagy in the maintenance of immunological memory against influenza infection," Nature Medicine, vol. 20, no. 5, pp. 503-510, 2014.

[82] K. Schlie, A. Westerback, L. DeVorkin et al., "Survival of effector $\mathrm{CD}^{+} \mathrm{T}$ cells during influenza infection is dependent on autophagy," Journal of Immunology, vol. 194, no. 9, pp. 42774286, 2015.

[83] G. Liu, S. Xiong, Y.-F. Xiang et al., "Antiviral activity and possible mechanisms of action of pentagalloylglucose (PGG) against influenza A virus," Archives of Virology, vol. 156, no. 8, pp. 1359-1369, 2011.

[84] G. Liu, M. Zhong, C. Guo et al., "Autophagy is involved in regulating influenza A virus RNA and protein synthesis associated with both modulation of Hsp90 induction and mTOR/p70S6K signaling pathway," The International Journal of Biochemistry \& Cell Biology, vol. 72, pp. 100-108, 2016.

[85] P. Matarrese, L. Nencioni, P. Checconi et al., "Pepstatin A alters host cell autophagic machinery and leads to a decrease in influenza A virus production," Journal of Cellular Physiology, vol. 226, no. 12, pp. 3368-3377, 2011.

[86] J.-P. Dai, W.-Z. Li, X.-F. Zhao et al., "A drug screening method based on the autophagy pathway and studies of the mechanism 
of evodiamine against influenza A virus," PLoS ONE, vol. 7, no. 8, Article ID e42706, 2012.

[87] J. Dai, G. Wang, W. Li et al., "High-throughput screening for anti-influenza A virus drugs and study of the mechanism of procyanidin on influenza A virus-induced autophagy," Journal of Biomolecular Screening, vol. 17, no. 5, pp. 605-617, 2012.

[88] Y. Yan, Z. Zou, Y. Sun et al., "Anti-malaria drug chloroquine is highly effective in treating avian influenza A H5N1 virus infection in an animal model," Cell Research, vol. 23, no. 2, pp. 300-302, 2013.

[89] J.-P. Dai, X.-F. Zhao, J. Zeng et al., "Drug screening for autophagy inhibitors based on the dissociation of Beclin1-Bcl2 complex using bifc technique and mechanism of eugenol on anti-influenza A virus activity," PLoS ONE, vol. 8, no. 4, Article ID e61026, 2013.

[90] H.-Y. Zhu, L. Han, X.-L. Shi et al., "Baicalin inhibits autophagy induced by influenza A virus H3N2," Antiviral Research, vol. 113, pp. 62-70, 2015.

[91] B. Yeganeh, S. Ghavami, A. L. Kroeker et al., "Suppression of influenza A virus replication in human lung epithelial cells by noncytotoxic concentrations bafilomycin A1," American Journal of Physiology — Lung Cellular and Molecular Physiology, vol. 308, no. 3, pp. L270-L286, 2015.

[92] A. H.-Y. Law, D. C.-W. Lee, K.-Y. Yuen, M. Peiris, and A. S.-Y. Lau, "Cellular response to influenza virus infection: a potential role for autophagy in CXCL10 and interferon-alpha induction," Cellular and Molecular Immunology, vol. 7, no. 4, pp. 263-270, 2010.

[93] T. Kawai and S. Akira, "Innate immune recognition of viral infection," Nature Immunology, vol. 7, no. 2, pp. 131-137, 2006.

[94] C.-S. Shi and J. H. Kehrl, "MyD88 and Trif target Beclin 1 to trigger autophagy in macrophages," The Journal of Biological Chemistry, vol. 283, no. 48, pp. 33175-33182, 2008.

[95] H. Pan, Y. Zhang, Z. Luo et al., "Autophagy mediates avian influenza $\mathrm{H} 5 \mathrm{~N} 1$ pseudotyped particle-induced lung inflammation through NF- $\kappa$ B and p38 MAPK signaling pathways," American Journal of Physiology - Lung Cellular and Molecular Physiology, vol. 306, no. 2, pp. L183-L195, 2014.

[96] P. Chang, S. V. Kuchipudi, K. H. Mellits et al., "Early apoptosis of porcine alveolar macrophages limits avian influenza virus replication and pro-inflammatory dysregulation," Scientific Reports, vol. 5, Article ID 17999, 2015.

[97] R. Weiss, J. Laengle, M. Sachet et al., "Interleukin-24 inhibits influenza A virus replication in vitro through induction of tolllike receptor 3 dependent apoptosis," Antiviral Research, vol.123, pp. 93-104, 2015.

[98] H. Everett and G. McFadden, "Apoptosis: an innate immune response to virus infection," Trends in Microbiology, vol. 7, no. 4, pp. 160-165, 1999.

[99] J. L. McAuley and J. A. McCullers, "Pro-inflammatory effects of H5N1 and 20th century pandemic influenza virus PB1-F2 proteins are mediated by macrophages," Influenza and other Respiratory Viruses, vol. 5, pp. 280-283, 2011.

[100] D. Zamarin, A. García-Sastre, X. Xiao, R. Wang, and P. Palese, "Influenza virus PB1-F2 protein induces cell death through mitochondrial ANT3 and VDAC1," PLoS Pathogens, vol. 1, no. 1, article e4, 2005.

[101] G.-W. Chen, C.-C. Yang, K.-C. Tsao et al., "Influenza A virus PB1-F2 gene in recent taiwanese isolates," Emerging Infectious Diseases, vol. 10, no. 4, pp. 630-636, 2004.
[102] T. Yoshizumi, T. Ichinohe, O. Sasaki et al., "Influenza A virus protein PB1-F2 translocates into mitochondria via Tom 40 channels and impairs innate immunity," Nature Communications, vol. 5, article 4713, 2014.

[103] L. A. Perrone, J. K. Plowden, A. García-Sastre, J. M. Katz, and T. M. Tumpey, "H5N1 and 1918 pandemic influenza virus infection results in early and excessive infiltration of macrophages and neutrophils in the lungs of mice," PLoS Pathogens, vol. 4, no. 8, Article ID e1000115, 2008.

[104] C. R. Baskin, H. Bielefeldt-Ohmann, T. M. Tumpey et al., "Early and sustained innate immune response defines pathology and death in nonhuman primates infected by highly pathogenic influenza virus," Proceedings of the National Academy of Sciences of the United States of America, vol. 106, no. 9, pp. 3455-3460, 2009.

[105] M. D. de Jong, C. P. Simmons, T. T. Thanh et al., "Fatal outcome of human influenza A (H5N1) is associated with high viral load and hypercytokinemia," Nature Medicine, vol. 12, no. 10, pp. 1203-1207, 2006.

[106] J. L. McAuley, M. D. Tate, C. J. MacKenzie-Kludas et al., "Activation of the NLRP3 inflammasome by IAV Virulence protein PB1-F2 contributes to severe pathophysiology and disease," PLoS Pathogens, vol. 9, no. 5, Article ID e1003392, 2013.

[107] A. L. Reis and J. W. McCauley, "The influenza virus protein PB1F2 interacts with IKK $\beta$ and modulates NF- $\kappa \mathrm{B}$ signalling," PLoS ONE, vol. 8, no. 5, article e63852, 2013.

[108] C. Chevalier, A. Al Bazzal, J. Vidic et al., "PB1-F2 influenza A virus protein adopts a $\beta$-sheet conformation and forms amyloid fibers in membrane environments," The Journal of Biological Chemistry, vol. 285, no. 17, pp. 13233-13243, 2010.

[109] T. A. Timofeeva, H. D. Klenk, and O. P. Zhirnov, "Identification of the protease-binding domain in the N-terminal region of influenza virus A matrix protein M1," Molecular Biology, vol. 35, no. 3, pp. 411-416, 2001.

[110] O. P. Zhirnov, A. L. Ksenofontov, S. G. Kuzmina, and H. D. Klenk, "Interaction of influenza A virus M1 matrix protein with caspases," Biochemistry, vol. 67, no. 5, pp. 534-539, 2002.

[111] S. J. Morris, G. E. Price, J. M. Barnett, S. A. Hiscox, H. Smith, and C. Sweet, "Role of neuraminidase in influenza virus-induced apoptosis," Journal of General Virology, vol. 80, no. 1, pp. 137$146,1999$.

[112] S. Tripathi, J. Batra, W. Cao et al., "Influenza A virus nucleoprotein induces apoptosis in human airway epithelial cells: implications of a novel interaction between nucleoprotein and host protein clusterin," Cell Death and Disease, vol. 4, no. 3, article e562, 2013.

[113] H. Ying, T. Z. Zaks, R.-F. Wang et al., "Cancer therapy using a self-replicating RNA vaccine," Nature Medicine, vol. 5, no. 7, pp. 823-827, 1999.

[114] S. Balachandran, C. N. Kim, W.-C. Yeh, T. W. Mak, K. Bhalla, and G. N. Barber, "Activation of the dsRNA-dependent protein kinase, PKR, induces apoptosis through FADD-mediated death signaling," The EMBO Journal, vol. 17, no. 23, pp. 6888-6902, 1998.

[115] K. Uetani, S. D. Der, M. Zamanian-Daryoush et al., "Central role of double-stranded RNA-activated protein kinase in microbial induction of nitric oxide synthase," The Journal of Immunology, vol. 165, no. 2, pp. 988-996, 2000.

[116] H. Kato, O. Takeuchi, S. Sato et al., "Differential roles of MDA5 and RIG-I helicases in the recognition of RNA viruses," Nature, vol. 441, no. 1, pp. 101-105, 2006. 
[117] W. J. Wurzer, C. Ehrhardt, S. Pleschka et al., "NF- $\kappa$ B-dependent induction of tumor necrosis factor-related apoptosis-inducing ligand (TRAIL) and Fas/FasL is crucial for efficient influenza virus propagation," The Journal of Biological Chemistry, vol. 279, no. 30, pp. 30931-30937, 2004.

[118] S. Herold, M. Steinmueller, W. von Wulffen et al., "Lung epithelial apoptosis in influenza virus pneumonia: the role of macrophage-expressed TNF-related apoptosis-inducing ligand," The Journal of Experimental Medicine, vol. 205, no. 13, pp. 3065-3077, 2008.

[119] B. Liu, D. Meng, T. Wei, S. Zhang, Y. Hu, and M. Wang, "Apoptosis and pro-inflammatory cytokine response of mast cells induced by influenza A viruses," PLoS ONE, vol. 9, no. 6, Article ID e100109, 2014.

[120] J. Jaworska, F. Coulombe, J. Downey et al., "NLRX1 prevents mitochondrial induced apoptosis and enhances macrophage antiviral immunity by interacting with influenza virus PB1-F2 protein," Proceedings of the National Academy of Sciences of the United States of America, vol. 111, no. 20, pp. E2110-E2119, 2014.

[121] A. T. Tran, M. N. Rahim, C. Ranadheera et al., "Knockdown of specific host factors protects against influenza virus-induced cell death," Cell Death and Disease, vol. 4, no. 8, article e769, 2013.

[122] K. Kosai, M. Seki, A. Tanaka et al., "Increase of apoptosis in a murine model for severe pneumococcal pneumonia during influenza A virus infection," Japanese Journal of Infectious Diseases, vol. 64, no. 6, pp. 451-457, 2011.

[123] M. C. Abt, L. C. Osborne, L. A. Monticelli et al., "Commensal bacteria calibrate the activation threshold of innate antiviral immunity," Immunity, vol. 37, no. 1, pp. 158-170, 2012.

[124] T. Ichinohe, I. K. Pang, Y. Kumamoto et al., "Microbiota regulates immune defense against respiratory tract influenza A virus infection," Proceedings of the National Academy of Sciences of the United States of America, vol. 108, no. 13, pp. 5354-5359, 2011.

[125] J. Wang, F. Li, R. Sun et al., "Bacterial colonization dampens influenza-mediated acute lung injury via induction of M2 alveolar macrophages," Nature Communications, vol. 4, article 2106, 2013.

[126] A. I. Wolf, M. C. Strauman, K. Mozdzanowska et al., "Pneumolysin expression by Streptococcus pneumoniae protects colonized mice from influenza virus-induced disease," Virology, vol. 462-463, no. 1, pp. 254-265, 2014.

[127] M. Miettinen, S. Matikainen, J. Vuopio-Varkila et al., "Lactobacilli and streptococci induce interleukin-12 (IL-12), IL-18, and gamma interferon production in human peripheral blood mononuclear cells," Infection and Immunity, vol. 66, no. 12, pp. 6058-6062, 1998.

[128] M. Nakamatsu, N. Yamamoto, M. Hatta et al., "Role of interferon- $\gamma$ in $\mathrm{V} \alpha 14+$ natural killer $\mathrm{T}$ cell-mediated host defense against Streptococcus pneumoniae infection in murine lungs," Microbes and Infection, vol. 9, no. 3, pp. 364-374, 2007.

[129] U. Koppe, K. Högner, J.-M. Doehn et al., "Streptococcus pneumoniae stimulates a STING- and IFN regulatory factor 3dependent type I IFN production in macrophages, which regulates RANTES production in macrophages, cocultured alveolar epithelial cells, and mouse lungs," Journal of Immunology, vol. 188, no. 2, pp. 811-817, 2012.

[130] T. L. McCool, T. R. Cate, G. Moy, and J. N. Weiser, "The immune response to pneumococcal proteins during experimental human carriage," The Journal of Experimental Medicine, vol. 195, no. 3, pp. 359-365, 2002.
[131] E. A. Joyce, S. J. Popper, and S. Falkow, "Streptococcus pneumoniae nasopharyngeal colonization induces type I interferons and interferon-induced gene expression," BMC Genomics, vol. 10, article 404, 2009.

[132] X. G. Guo, S. Zhou, and Y. Xia, "Autophagy is a defense mechanism in the infection of lung epithelial celss for streptococcus pneumonia," in Respirology, p. 165, Wiley-Blackwell, Hoboken, NJ, USA, 2013.

[133] Y. Xu, C. Jagannath, X.-D. Liu, A. Sharafkhaneh, K. E. Kolodziejska, and N. T. Eissa, "Toll-like receptor 4 is a sensor for autophagy associated with innate immunity," Immunity, vol. 27, no. 1, pp. 135-144, 2007.

[134] A. Amano, I. Nakagawa, and T. Yoshimori, "Autophagy in innate immunity against intracellular bacteria," Journal of Biochemistry, vol. 140, no. 2, pp. 161-166, 2006.

[135] P. Li, J. Shi, Q. He et al., "Streptococcus pneumoniae induces autophagy through the inhibition of the PI3K-I/Akt/mTOR pathway and ROS hypergeneration in A549 cells," PLoS ONE, vol. 10, no. 3, Article ID e0122753, 2015.

[136] K. Ouyang, S. A. Woodiga, V. Dwivedi et al., "Pretreatment of epithelial cells with live Streptococcus pneumoniae has no detectable effect on influenza A virus replication In vitro," PLoS ONE, vol. 9, Article ID e90066, 2014.

[137] L. Fang, H.-M. Wu, P.-S. Ding, and R.-Y. Liu, “TLR2 mediates phagocytosis and autophagy through JNK signaling pathway in Staphylococcus aureus-stimulated RAW264.7 cells," Cellular Signalling, vol. 26, no. 4, pp. 806-814, 2014.

[138] I. Christopoulou, K. Roose, L. I. Ibañez, and X. Saelens, "Influenza vaccines to control influenza-associated bacterial infection: where do we stand?" Expert Review of Vaccines, vol. 14, no. 1, pp. 55-67, 2014.

[139] B. Michiels, K. van Puyenbroeck, V. Verhoeven, E. Vermeire, and S. Coenen, "The value of neuraminidase inhibitors for the prevention and treatment of seasonal influenza: a systematic review of systematic reviews," PLoS ONE, vol. 8, no. 4, Article ID e60348, 2013.

[140] J. J. Treanor, F. G. Hayden, P. S. Vrooman et al., "Efficacy and safety of the oral neuraminidase inhibitor oseltamivir in treating acute influenza: a randomized controlled trial," Journal of the American Medical Association, vol. 283, no. 8, pp. 1016$1024,2000$.

[141] J. A. McCullers, "Influenza," in Textbook of Clinical Pediatrics, pp. 1199-1208, 2012.

[142] F. Carrat and A. Flahault, "Influenza vaccine: the challenge of antigenic drift," Vaccine, vol. 25, no. 39-40, pp. 6852-6862, 2007.

[143] H. W. Boucher and G. R. Corey, "Epidemiology of methicillinresistant Staphylococcus aureus," Clinical Infectious Diseases, vol. 46, no. 5, pp. S344-S349, 2008.

[144] B. K. Giersing, S. S. Dastgheyb, K. Modjarrad, and V. Moorthy, "Status of vaccine research and development of vaccines for Staphylococcus aureus," Vaccine, 2016.

[145] D. R. Hahn, C.-L. Na, and T. E. Weaver, "Reserve autophagic capacity in alveolar epithelia provides a replicative niche for influenza A virus," American Journal of Respiratory Cell and Molecular Biology, vol. 51, no. 3, pp. 400-412, 2014. 

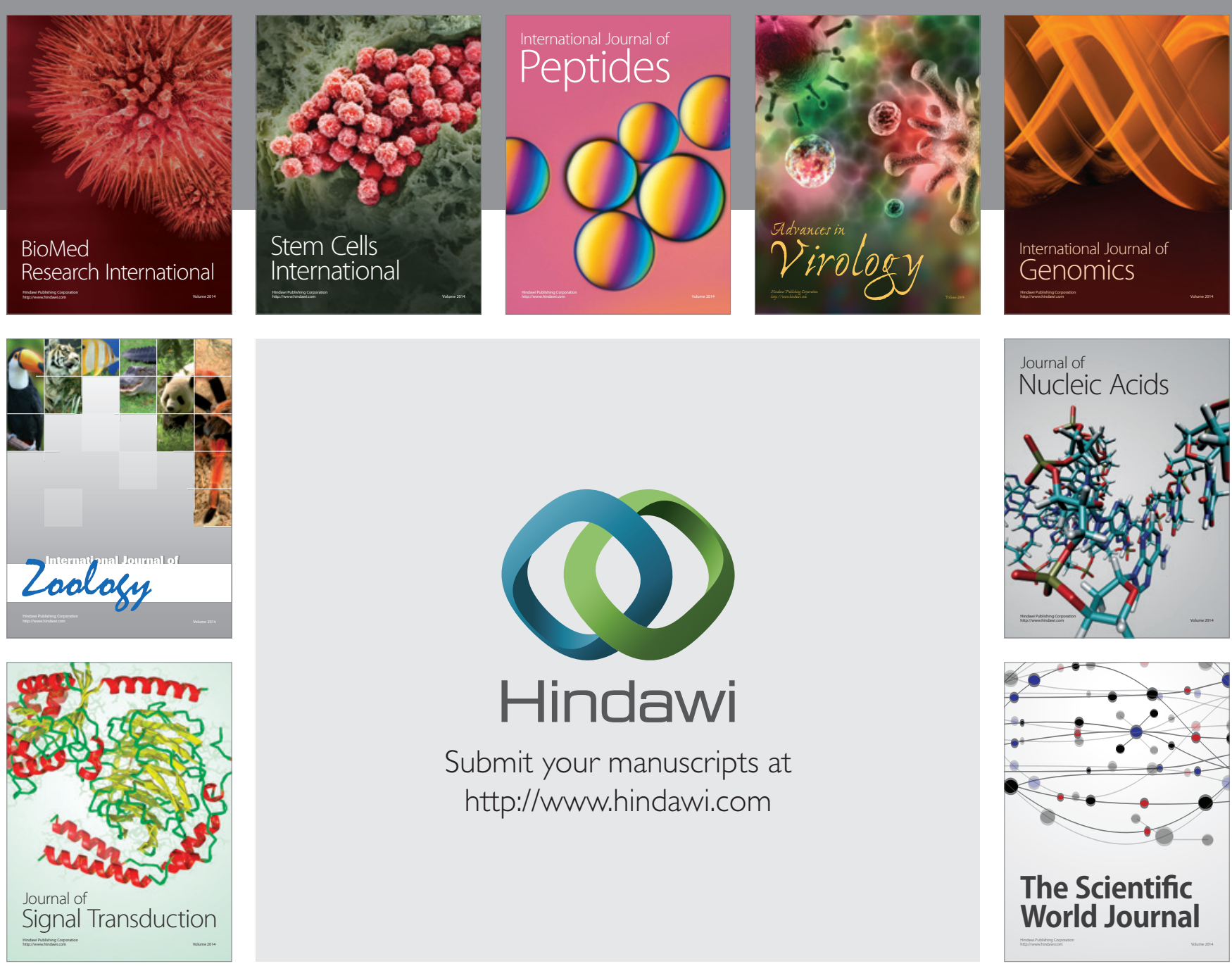

Submit your manuscripts at

http://www.hindawi.com
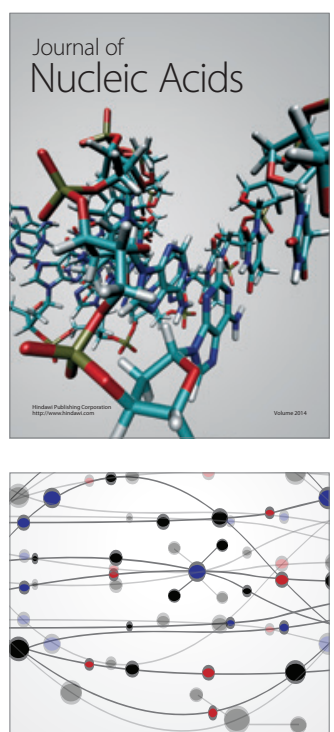

The Scientific World Journal
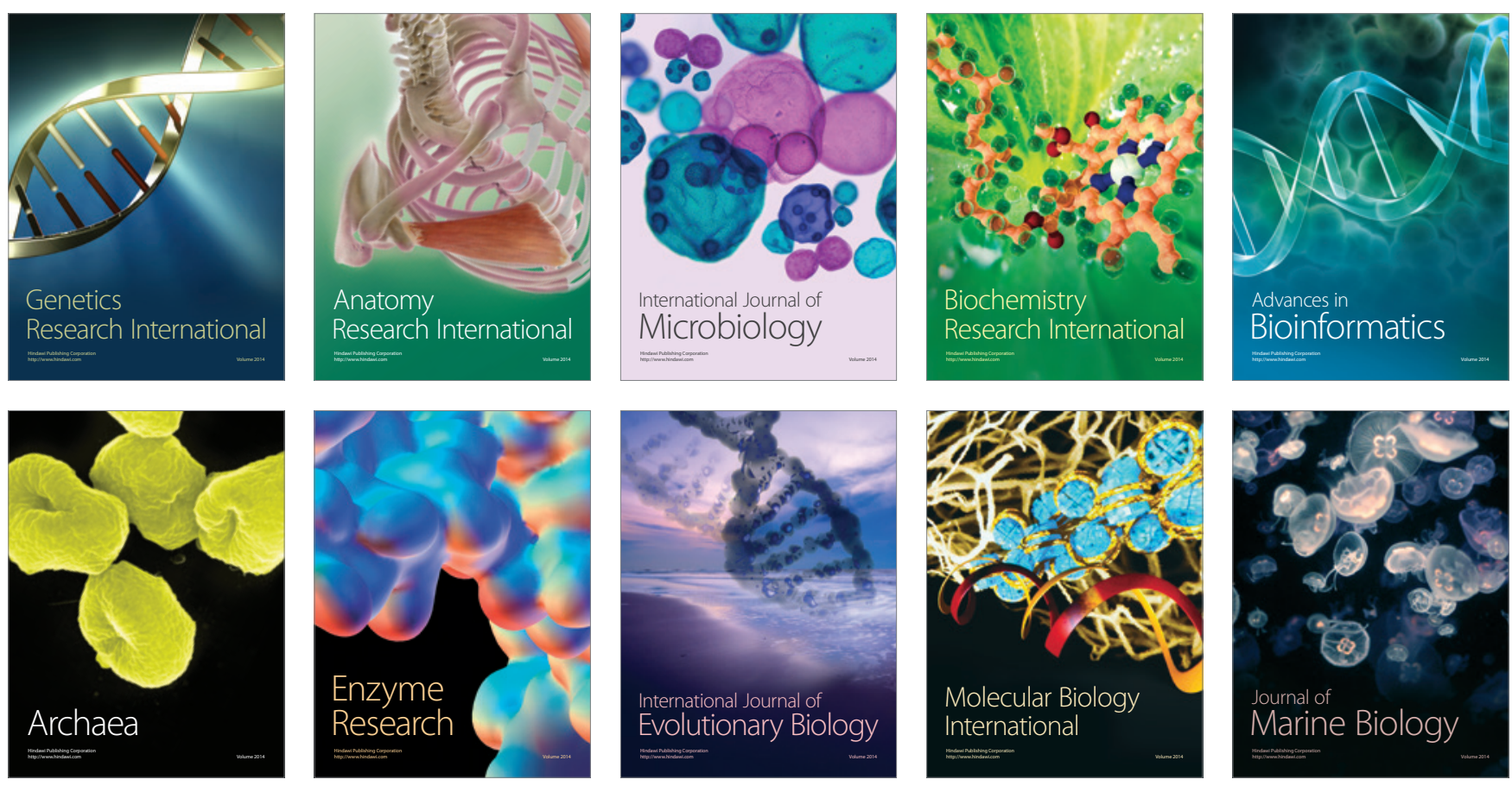